\title{
Viral infections and atherothrombosis: Another caution in the wake of COVID-19?
}

\author{
(D) Marcel de Paula Pereira ${ }^{1}$ \\ (iD) Eduardo Gomes Lima ${ }^{1}$ \\ Carlos Vicente Serrano Junior ${ }^{1}$
}

1. Departamento de Aterosclerose - Instituto do Coração (InCor) - HCFMUSP, São Paulo, SP, Brasil.

\section{INTRODUCTION}

The COVID-19 pandemic is caused by the novel coronavirus (Sars-COV-2), with global impact and leading to numerous hospitalizations and deaths in several countries. Data from 01 April 2020 show over 887,000 infected individuals and over 44,000 deaths.

Publications ${ }^{1-4}$ show that the main risk factors for an unfavorable outcome are: hypertension 6-31\% (SAH), diabetes 7.3-19\% (DM), age, and presence of cardiovascular disease $8-15 \%$ (CVD); the main risk factor to date is age over 80 years. It should be noted, therefore, that patients with cardiovascular disease are at higher risk of complications. COVID-19 invades cells by binding to the of the angiotensin-converting enzyme (ACE-2), which has a greater expression in cardiac and pulmonary tissues ${ }^{5}$.

Zhou, et al., ${ }^{2}$ has reported the clinical characteristics of 171 patients admitted with infections caused by COVID-19. Among the abnormal laboratory results found in multivariate analysis was the increase of D-dimer $>1 \mathrm{ug} / \mathrm{ml}$, which was related to worse prognosis, as were advanced age and increased SOFA score. Studies have also demonstrated that many patients can evolve with lymphopenia ${ }^{1.4}$. The presence of coagulopathy, evidenced by the increase of D-dimer, prothrombin time (PT), and disseminated intravascular coagulation (DIC), was also a marker of negative prognosis, regardless of its high levels during admission or at any time during hospitalization ${ }^{2.6}$. In a recent meta-analysis ${ }^{7}$, high levels of troponin were also related to a worse prognosis.

In another publication on 416 hospitalized patients ${ }^{8}$, there was an association between increased troponin and mortality; whereas the higher its value, the higher was the risk. Myocardial injury was identified in $19.7 \%$ of patients. These were older, had a higher number of comorbidities (including cardiovascular), and presented other increased inflammatory parameters in relation to the control group, such as increased leukocytes, C-reactive protein, and procalcitonin, as well as greater opacity on computed tomography scans of the chest. In addition, these patients had a longer period of mechanical 
ventilation, renal dysfunction, and coagulation disorders. The difference in mortality was $51.2 \%$ vs. $4.5 \%$ $(\mathrm{P}<0.001)$. This inflammatory storm, with increased cytokines, troponin, D-dimer, among others, leads to a greater predisposition to cardiovascular events and death. In addition, data show that acute coronary events, arrhythmias, myocarditis, Takotsubo syndrome, and acute heart failure can occur related to COVID-19. In relation to acute heart complications, Wang ${ }^{4}$ reported $16 \%$ of arrhythmia.

Preliminary evidence ${ }^{9-12}$ demonstrates that infections, viral ones included, lead to systemic inflammatory conditions, including atheromatous plaques. As shown in Figure 1, during the inflammatory activity triggered by the infection, there is an infiltrate of cells in the atheromatous plaque ( $\mathrm{T}$ cells, macrophages, and neutrophils), contributing to the production of cytokines, coagulation factors, oxygen radicals, and vasoactive molecules, leading to increased vascular permeability, endothelial damage, rupture of the fibrous layer, and exposure of thrombogenic elements (collagen, tissue factor, and platelet adhesion molecules), contributing to the formation of thrombus. The plaque erosion alone is not enough to cause coronary ischemic syndromes. Other important factors for the emergence of injury and ischemia are: the nature of the thrombus formation (partial vs. total), degree of stenosis, presence of vasoconstriction, distal perfusion, and an imbalance between supply and demand in the coronary bed evidenced by hypoxia, hypotension, tachycardia, and the increase of endogenous catecholamines leading to local vasoconstriction. Previous reviews ${ }^{13-15}$ have demonstrated the role of platelets and the coagulation system in viral infections. It is suggested that platelets can bind to pathogens through receptors on their surface and help eradicate them. After the binding, there is a release of factors such as Thromboxane A2 and Adenosine Diphosphate (ADP), activating the coagulation and inflammatory cascade. There have been reports that this platelet activation results in thrombocytopenia, a common finding in viral infections (such as Influenza, Coronaviruses, and HIV). An endothelial lesion can lead to exposure of prothrombotic factors, such as the Von Willebrand factor, on the vessel wall, as well as to the decrease of endogenous anticoagulant factors, such as proteins $\mathrm{C}$ and $\mathrm{S}$, and antithrombin. Previous data show that in infections caused by Influenza and SARS, there was

FIGURE 1. ACUTE INFECTIONS TRIGGERING MYOCARDIAL INJURY AND ACUTE CORONARY SYNDROMES. (ADAPTED FROM MEDINA ET, AL; ROLE OF ACUTE INFECTION IN TRIGGERING ACUTE CORONARY SYNDROMES; LANCET INFECT DIS 2010; 10: 83-92)

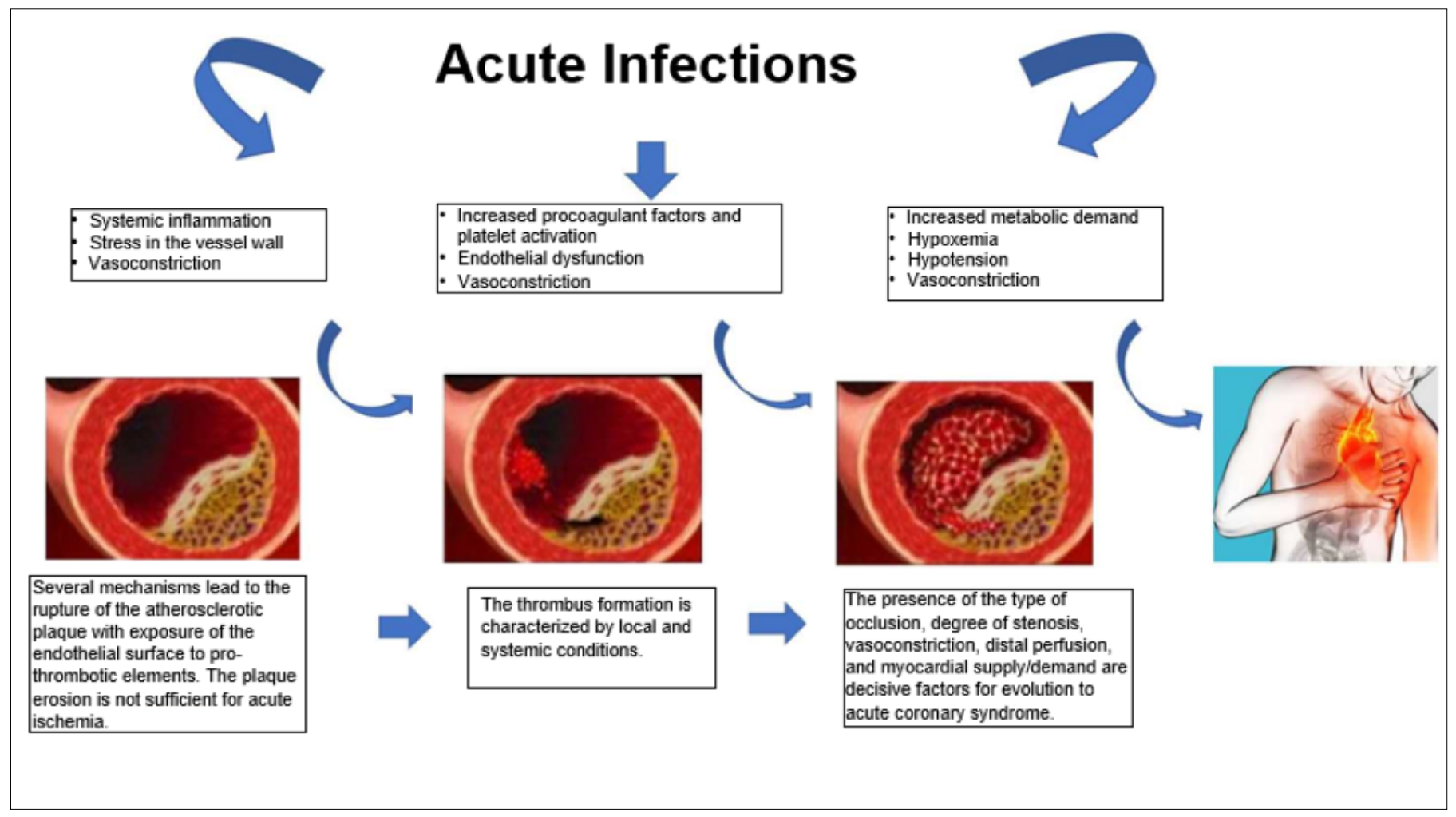


an increase of thrombi and fibrin deposits in the lungs, thus suggesting local microthrombosis.

There have also been descriptions of "shock-induced Endotheliopathy in critical patients"12, which suggests that during shock there is sympathetic -adrenal hyperactivation, with an increase of circulating catecholamines, leading to endothelial and microcirculation damage due to capillary leak, with increased local inflammatory mediators, vascular edema, and tissue hypoxia, generating a vicious cycle of endothelial lesion. Thus, the endogenous anticoagulant system would be damaged, leading to microvascular occlusion, which can justify the increased D-dimer and troponin in more severe patients. In addition, in the final shock cascade, there can be coagulopathy and even DIC as a compensatory mechanism. Johansson et al. suggested that young people have less endotheliopathy (in patients with trauma-related shock) in comparison with the elderly. In addition, smokers, hypertensive, and diabetics patients have greater endothelial dysfunction, and the finding of endotheliopathy could be associated with the outcomes of death in the short and long term.

In a study published in $2018^{16}$, viral infections by Influenza were associated with a higher rate of acute coronary events. It evaluated the diagnoses of acute myocardial infarction (AMI) during a 1-year period before and after a positive diagnosis for Influenza. In this study, there was a higher rate of AMI in up to 7 days after the diagnosis of Influenza in relation to the control group. According to the data presented, infections, among them, viral ones included, can lead to acute coronary conditions, as well as endothelial and microvascular lesions. Increased D-dimer and troponin suggest a worse prognosis and greater risk of mortality in COVID-19 positive patients, probably because they are associated with a greater inflammatory response, endothelial damage, and thrombotic events.

A Chinese study ${ }^{17}$ that included 449 patients admitted for COVID-19 showed that the strategy of prescribing enoxaparin 40-60mg/day or non-fractionated heparin 10,000 to $15.000 \mathrm{U} /$ day brought benefits in mortality after 28 days in 2 subgroups. One of them comprised patients with criteria of coagulopathy induced by sepsis $>=4$ (which uses the criteria of increase of PT, decreased platelet count, and increased SOFA score), with a difference of $40 \% \mathrm{vs}$. $64.2 \%(\mathrm{P}=0.029)$. The other subgroup was composed of patients with D-dimer $>6 x$ the upper threshold of normality, with a difference of $32.8 \%$ vs. $52.4 \%$ (P = 0.017), demonstrating that the strategy of prescribing chemical prophylaxis for venous thromboembolism (VTE) should be considered in patients admitted with COVID-19, even if they present low/intermediate risk of VTE.

In relation to the therapeutic procedures for patients with CVD who are infected with COVID-19, the hypothesis has been raised that medications that interfere with the ACE can be harmful to the patient. Since there have been no studies demonstrating such association, most medical societies recommend not discontinuing angiotensin-converting enzyme (ACE) inhibitors and angiotensin II receptor blockers (ARB), as well as any medications that were already in use, particularly those used with prognostic benefits ${ }^{5.18}$. Pro-thrombotic events, including acute coronary events, require increased attention, and there is no evidence that recommends the routine interruption of antiplatelet and anticoagulant drugs. In addition, the recommendations for influenza and pneumococcus vaccinations in patients with previous indications are maintained.

\section{Contribution of the authors}

a) Marcel de Paula Pereira: Author of the paper and literature review

b) Eduardo Gomes Lima: Paper concept and design

c) Carlos Vicente Serrano Junior: Paper concept and design and final approval

\section{REFERENCES}

1. Guan W, Ni Z, Hu Y et al. Clinical Characteristics of Coronavirus Disease 2019 in China. DOI: 10.1056/NEJMoa2002032

2. Fei Zhou, Ting Yu, Ronghui Du, Guohui Fan, et.atl; Clinical course and risk factors for mortality of adult inpatients with COVID-19 in Wuhan, China: a retrospective cohort study; https://doi.org/10.1016/S0140-6736(20)30566-3
3. Bo Li1, Jing Yang, Faming Zhao, et.al; Prevalence and impact of cardiovascular metabolic diseases on COVID-19 in China; Clinical Research in Cardiology; https://doi.org/10.1007/s00392-020-01626-9

4. Wang D, Hu B, Hu C, et al.Clinical Characteristics of 138 Hospitalized Patients with2019 Novel Coronavirus-Infected Pneumonia in Wuhan, China. JAMA. Published online February 07, 2020. doi:10.1001/jama.2020.1585 
5. Clerkin et al.; COVID-19 and Cardiovascular Disease; 10.1161/ CIRCULATIONAHA.120.046941

6. Tang, Ning et.al; Abnormal coagulation arameters are associated with poor prognosis in patients with novel coronavirus pneumonia; J Thromb Haemost. 2020;00:1-4.

7. Cardiac troponin I in patients with coronavirus disease 2019 (COVID-19): Evidence from a meta-analysis; https://doi.org/10.1016/j.pcad.2020.03.001

8. Shaobo Shi, et.al; Association of Cardiac Injury With Mortality in Hospitalized Patients With COVID-19 inWuhan, China; JAMA Cardiol. doi:10.1001/ jamacardio.2020.0950

9. Braunwald's Heart Disease Review and Assessment, 11edition, 2018

10. Vicente F Corrales-Medina, Mohammad Madjid, Daniel M Musher; Role of acute infection in triggering acute coronary syndromes; Lancet Infect Dis 2010; 10: 83-92

11. Higuchi et al; Mycoplasma and chlamydia in vulnerable plaques associated to AMI; Arq Bras Cardiol, volume $81\left(n^{\circ} 1\right), 12-22,2003$
12. Johansson et al; Shock induced endotheliopathy (SHINE) in acute critical illness - a unifying pathophysiologic mechanism; Critical Care (2017) 21:25

13. Seyoum et.al; Human blood platelets and viruses: defense mechanism and role in the removal of viral pathogens; Thrombosis Journal (2018) 16:16

14. Antoniak, Silvio; The coagulation system in host defense; Res Pract Thromb Haemost. 2018;2:549-55

15. Goeijenbier et.al; Review: Viral Infections and Mechanisms of Thrombosis and Bleeding; Journal of Medical Virology 84:1680-1696 (2012)

16. Jeffrey C. Kwong, M.D., Kevin L. Schwartz, M.D., Michael A. Campitelli, M.P.H, et.al; Acute Myocardial Infarction after Laboratory-Confirmed Influenza Infection; N Engl J Med 2018;378:345-53.

17. Tang, Ning et.at; Anticoagulant treatment is associated with decreased mortality in severe coronavirus disease 2019 patients with coagulopathy; doi: 10.1111/ JTH.14817

18. Vaduganathan, Muthiah, Renin-Angiotensin-Aldosterone System Inhibitors in Patients with Covid-19; DOI: 10.1056/NE|Msr2005760 